\title{
Partial but Rapid Recovery from Paralysis after Immunomodulation during Early Stage of Neuralgic Amyotrophy
}

\author{
Masashi Nakajima Shinsuke Fujioka Hideki Ohno Kohnosuke Iwamoto \\ Department of Neurology, Tokyo Rosai Occupational Diseases and Injuries Hospital, Tokyo, Japan
}

Dear Sir,

Neuralgic amyotrophy is a well-established clinical syndrome characterized by an acute onset of shoulder and arm pain followed by muscular weakness, wasting and variable sensory loss [1]. Most neurophysiological studies have reported features of axonal degeneration [2], reflecting terminal pathological results rather than the underlying process. We report a patient with neuralgic amyotrophy who was examined within a week after pain onset. The investigative results suggested that, in addition to axonal injury, reversible dysfunction within the brachial plexus played a part in the acute profound weakness.

\section{Case Report}

A previously healthy 39-year-old male mechanic awoke suddenly with intense left shoulder pain that lasted more than $24 \mathrm{~h}$. Paralysis of the left fingers developed the next morning. He visited an orthopedic clinic and underwent magnetic resonance imaging of the cervical spine and brachial plexus, which detected no abnormalities. His family history was unremarkable. A neurological examination on the sixth day after the onset of left shoulder pain (day 6) detected an isolated motor deficit in his left upper limb. The finger extensors and ulnar nerve-innervated hand muscles were completely paralyzed. Disabling weakness
[Medical Research Council (MRC) 2-3] was detected in the triceps brachii, flexor carpi ulnaris and median nerve-innervated hand muscles. The flexor carpi radialis and ulnaris, extensor carpi ulnaris and finger flexors were mildly impaired. The shoulder girdle muscles, the biceps brachii, brachioradialis and extensor carpi radialis were spared. The muscle stretch reflexes were normal, except for a diminished left triceps jerk. There was no sensory deficit in the left upper limb. The routine blood test results were normal. The negative results included antiganglioside IgG and IgM antibodies, antinuclear antibodies, antineutrophil cytoplasmic antibodies, HBs antigens and serum $\mathrm{M}$ proteins. Cerebrospinal fluid examination showed a normal cell count and protein content. A 3-day course of intravenous methylprednisolone (20 mg/kg daily) was prescribed, but there were no changes the next day. The patient underwent a 5day intravenous immunoglobulin course ( $0.4 \mathrm{~g} / \mathrm{kg}$ daily) from days 7 through 11 . Motor strength gradually returned during the regimen. On day 12 he could hold, pick up and release objects with his left hand, which had been useless before treatment. Although moderate left shoulder pain persisted, he went back to work on day 15 . On follow-up examinations on day 54 his mo- tor weakness had not changed, and the forearm extensors and ulnar-innervated hand muscles had wasted.

Nerve conduction studies were performed on day 7 before starting the intravenous immunoglobulin course. After stimulation up to Erb's point evoked compound muscle action potentials (CMAPs) were normal for the left extensor indicis proprius, abductor digiti minimi and abductor pollicis brevis despite complete or profound paralysis of these muscles, as compared to those of contralateral homonymous muscles, which had normal strength (table 1). The motor conduction velocities and minimum $\mathrm{F}$ wave latencies were normal, whereas the $\mathrm{F}$ wave persistence in the abductor pollicis brevis muscle was less after left than after right median nerve stimulation (table 1). The left abductor digiti minimi showed a late response of constant latency and waveform that most likely was an axon reflex [3], and $\mathrm{F}$ waves seemed to be absent (fig. 1B). On needle electromyography in the left extensor digitorum communis, only one motor unit was recruited, which had a high firing rate of about $50-60 \mathrm{~Hz}$. Immediately after intravenous immunoglobulin treatment (day 12) the abductor pollicis brevis muscle regained a strength of MRC 4 , and the $\mathrm{F}$ wave persistence was full (fig. 1C). In the

\section{KARGER}

Fax +41 613061234 E-Mail karger@karger.ch www.karger.com
(C) 2006 S. Karger AG, Base 0014-3022/06/0554-0227\$23.50/0

Accessible online at: www.karger.com/ene
Dr. Masashi Nakajima

Department of Neurology

Tokyo Rosai Occupational Diseases and Injuries Hospital

4-13-21 Ohmori-minami, Ohta-ku, Tokyo 143-0013 (Japan)

Tel. +8133742 7301, Fax +8133744 9310, E-mail masashi@tokyoh.rofuku.go.jp 
Table 1. Compound muscle action potential amplitudes and $\mathrm{F}$ wave parameters

\begin{tabular}{|c|c|c|c|c|c|c|}
\hline Nerve & Site $^{\mathrm{a}}$ & Day 7 & Day 12 & Day 19 & Day 34 & Day 54 \\
\hline \multicolumn{7}{|l|}{$L$ median } \\
\hline \multirow[t]{4}{*}{ CMAP, mV } & wrist & 9.0 & 8.0 & 7.8 & 6.5 & 6.5 \\
\hline & elbow & 9.0 & 7.8 & 7.5 & 6.3 & 6.3 \\
\hline & axilla & 8.9 & 7.5 & 7.0 & 6.3 & 6.0 \\
\hline & Erb & 8.6 & - & - & 6.6 & 6.1 \\
\hline \multicolumn{7}{|l|}{ F wave } \\
\hline Latency $^{\mathrm{b}}$ & & 28.9 & 29.2 & 28.4 & 27.8 & 28.8 \\
\hline Persistence ${ }^{c}$ & & $4 / 16$ & $16 / 16$ & $16 / 16$ & $7 / 16$ & $8 / 16$ \\
\hline \multicolumn{7}{|l|}{ L ulnar } \\
\hline \multirow[t]{4}{*}{ CMAP, mV } & wrist & 5.7 & 3.0 & 1.6 & 1.6 & 2.6 \\
\hline & elbow & 6.6 & 3.0 & 1.7 & 1.6 & 2.5 \\
\hline & axilla & 5.1 & 2.8 & 1.4 & 1.3 & 1.8 \\
\hline & Erb & 4.5 & 2.2 & - & 1.0 & 1.7 \\
\hline \multicolumn{7}{|l|}{ F wave } \\
\hline Latency & & n.e. & 31.0 & 29.7 & 33.6 & 36.2 \\
\hline Persistence & & $0 / 16$ & $11 / 16$ & $7 / 16$ & 716 & $2 / 16$ \\
\hline \multicolumn{7}{|l|}{ L radial } \\
\hline \multirow[t]{3}{*}{ CMAP, mV } & forearm & 3.5 & 1.2 & - & 1.2 & 0.5 \\
\hline & elbow & 3.2 & 1.1 & - & 1.1 & 0.3 \\
\hline & Erb & 3.0 & 1.0 & - & 0.5 & 0.3 \\
\hline \multicolumn{7}{|l|}{$R$ median } \\
\hline \multirow[t]{3}{*}{ CMAP, mV } & wrist & 10.1 & - & 10.1 & - & 8.2 \\
\hline & elbow & 10.1 & - & 10.0 & - & 8.2 \\
\hline & axilla & 10.0 & - & - & - & - \\
\hline \multicolumn{7}{|l|}{ F wave } \\
\hline Latency & & 27.4 & - & 28.1 & - & 29.3 \\
\hline Persistence & & $11 / 16$ & - & $12 / 16$ & - & $7 / 16$ \\
\hline \multicolumn{7}{|l|}{ Rulnar } \\
\hline \multirow[t]{3}{*}{ CMAP, mV } & wrist & 6.9 & - & 7.3 & - & 7.5 \\
\hline & elbow & 6.3 & - & 6.6 & - & 7.2 \\
\hline & axilla & 5.8 & - & 6.4 & - & 6.5 \\
\hline \multicolumn{7}{|l|}{ F wave } \\
\hline Latency & & 29.1 & - & 27.1 & - & 28.5 \\
\hline Persistence & & $16 / 16$ & - & $14 / 16$ & - & $16 / 16$ \\
\hline \multicolumn{7}{|l|}{$R$ radial } \\
\hline \multirow[t]{3}{*}{ CMAP, mV } & forearm & 2.7 & - & - & - & - \\
\hline & elbow & 2.2 & - & - & - & - \\
\hline & Erb & 2.2 & - & - & - & - \\
\hline \multicolumn{7}{|c|}{$\begin{array}{l}\text { CMAP: negative peak amplitude of the CMAP. } \mathrm{L}=\mathrm{Left} ; \mathrm{R}=\text { right; }-=\text { not done; } \\
\text { n.e. = not evoked. } \\
\text { a Site of stimulation. } \\
{ }^{\mathrm{b}} \text { Minimum F wave latency (ms). } \\
{ }^{\mathrm{c}} \text { Number of F waves clearly identified in } 16 \text { trials. }\end{array}$} \\
\hline
\end{tabular}

abductor digiti minimi muscle trace movement (MRC 2) and at least 4 different types of $F$ wave had returned (fig. 1D). The CMAP amplitudes, however, decreased in the previously paralyzed muscles during subsequent testing (table 1). Needle elec- tromyography showed fibrillations and positive sharp waves in the left extensor indicis proprius and flexor carpi radialis. Except for one short run of fibrillation at the C7-T1 intervertebral level, there was no acute denervation in the left cervical paraspinal muscles. The sensory nerve action potential amplitudes and conduction velocities in the median, ulnar and superficial radial nerves were normal. 
Fig. 1. F waves after left median $(\mathbf{A}, \mathbf{C})$ and ulnar (B, D) stimulation at the wrist. In the left median nerve the number of $F$ waves is increased after treatment $(\mathbf{C})$ as compared to before treatment (A). B The left ulnar nerve shows a late response at $27.9 \mathrm{~ms}$ with a consistent latency and waveform (axon reflex) before treatment. D After treatment 11 late responses consisting of 4 different types of waveforms have appeared. The horizontal and vertical scales are identical in all the recordings.

\section{Discussion}

We had the chance to examine this patient with distal muscle weakness very early in the disease process. The CMAPs in the above muscles were normally evoked after stimulation up to Erb's point. Therefore, with respect to profound motor weakness during this early stage, the responsible lesion should be located proximal to Erb's point. The patient's incomplete but rapid recovery from paralysis, regardless of whether it is spontaneous or induced by immunomodulating agents, is unusual in neuralgic amyotrophy and contradicts its pure axonal pathology. The paucity or absence of $\mathrm{F}$ waves, together with their reappearance associated with the partial recovery of strength of the corresponding muscles, could be indirect signs of a conduction block and its partial resolution [3]. Coexistent acute axonal injury during this early stage, on the other hand, is also evident based on the significantly reduced CMAP amplitudes and acute denervation in the affected limb on day 12 after pain onset.

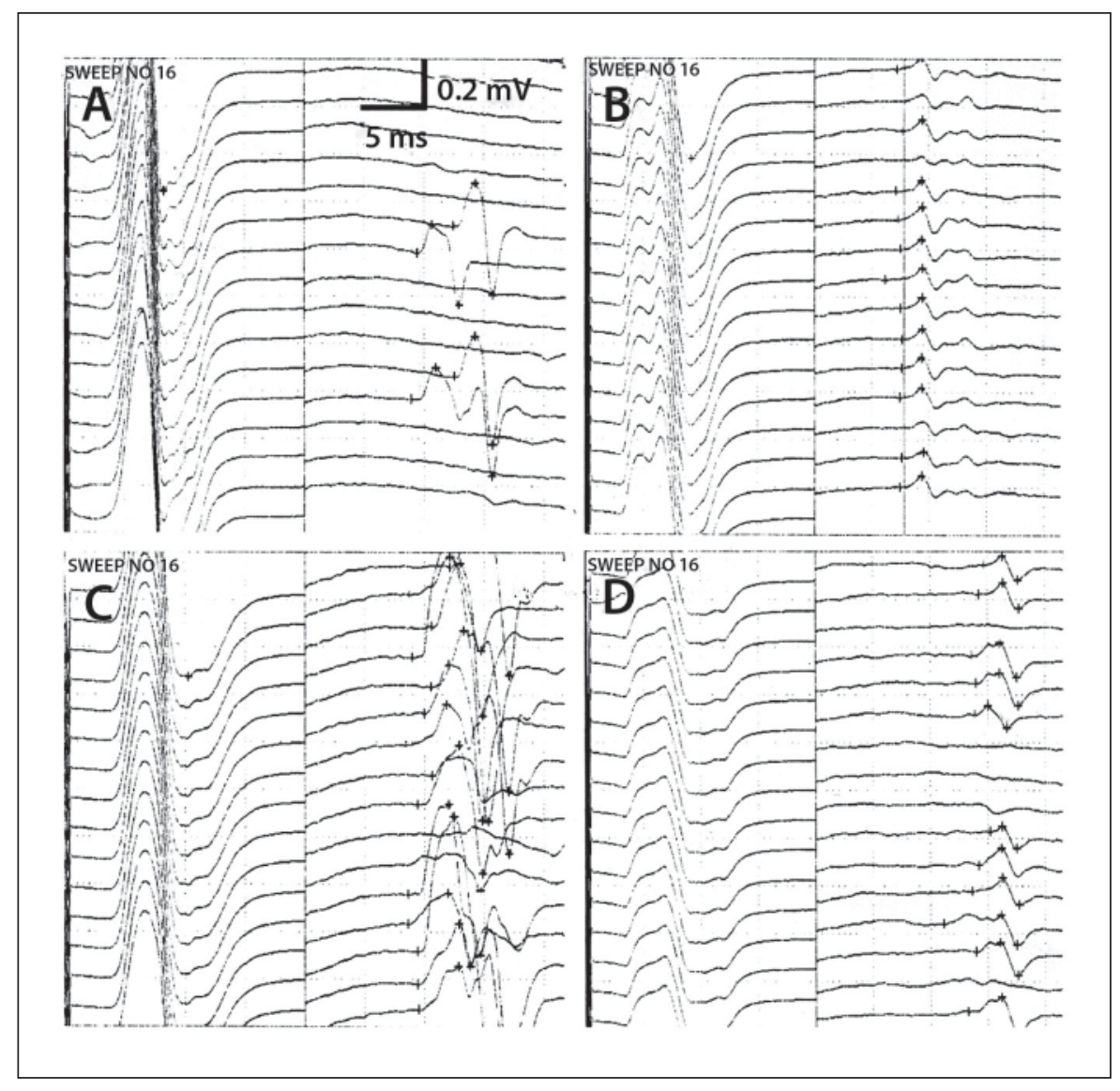

In a few cases of neuralgic amyotrophy evidence of proximal motor conduction block has been provided [2,4]. Watson et al. [4] showed a partial conduction block between the cervical spinal roots and axilla in 2 patients $6-8$ weeks after onset. It was resolved 6 weeks later and was not accompanied by a reduction in distal CMAP amplitudes. As compared to their cases our patient had a much more pronounced weakness followed by axonal degeneration, features known to be typical of neuralgic amyotrophy. We posit that an early examination, made within days of the onset of pain, may detect the focal underlying pathology in greater numbers of patients suffering from neuralgic amyotrophy. Recovery from neuralgic amyotrophy depends on the severity of weakness during the acute phase, which may begin very late in the illness [5], even taking years in the distal muscles [6]. The case of our patient illustrates the possibility that therapeutic intervention during the early stage of neuralgic amyotrophy minimizes and shortens functional impairment.

\section{Acknowledgements}

We thank Dr. Jun Shimizu and Dr. Satoko Kano for the antiganglioside antibody assays.

\section{References}

1 Parsonage MJ, Turner JWA: Neuralgic amyotrophy: the shoulder girdle syndrome. Lancet 1948;i:973-978.

2 Lo Y-L, Mills KR: Motor root conduction in neuralgic amyotrophy: evidence of proximal conduction block. J Neurol Neurosurg Psychiatry 1999;66:586-590.

3 Kimura J: Electrodiagnosis in Diseases of Nerve and Muscle. Principles and Practice, ed 2. Philadelphia, Davis, 1989.

4 Watson BV, Nicolle MW, Brown JD: Conduction block in neuralgic amyotrophy. Muscle Nerve 2001;24:559-563.

5 Dixon GJ, Dick TBS: Acute brachial radiculitis: course and prognosis. Lancet 1945;ii: 707-708.

6 Tsairis P, Dyck PJ, Mulder DW: Natural history of brachial plexus neuropathy: report on 99 cases. Arch Neurol 1972;27:109-117. 